

\title{
Creating a "SafeSpot" for mental health education in schools
}

\author{
By Dr. Jessica K Edwards
}

SafeSpot is a digital educational programme that aims to raise awareness of mental health problems and develop helpful coping strategies to improve mental health in young people. The SafeSpot programme combines classroom tutorials delivered by teachers, with a website and a mobile phone app used by pupils, and peer support. Researchers have trialled SafeSpot with 2,320 pupils aged 11-14 years and 90 teachers from six secondary schools in Scotland. Now, Mallika Punukollu and colleagues have conducted qualitative focus groups with 31 of these pupils and 30 of these teachers to investigate their perceptions of SafeSpot.

"The SafeSpot mental health app and website is regularly used with young people in CAMHS in Glasgow, and is very positively received", explains Punukollu. "In this study, teachers and students also gave positive feedback about the SafeSpot educational mental health curriculum: it normalised the conversation about mental health in school settings and made it easier to access relevant resources and request assistance when needed". Despite these positive findings, participants thought that certain changes could improve the SafeSpot programme. For example, teachers reported mixed levels of pupil engagement with the SafeSpot resources and proposed that time should be designated to using the app in the classroom setting to improve engagement.

Further work is now needed to evaluate the cost-effectiveness of SafeSpot on the mental health of pupils over a longer period of time and to determine which elements of SafeSpot in particular are most effective. "It is clear that mental health education training should be provided in all schools", says Dr. Punukollu. "Based on our findings thus far, and in line with the government recommendations for all schools to have mental health education in the curriculum, SafeSpot could be suitable to roll out to schools".

\section{Referring to:}

Punukollu, M., Leighton, E.L., Brooks, A.F., Heron, S., Mitchell, F., Regener, P., Karagiorgou, O., Bell, C., Gilmour, M., Moya, N., Sharpe, H. \& Minnis, $H$. (2020), SafeSpot: an innovative app and mental health support package for Scottish schools - a qualitative analysis as part of a mixed methods study. Child Adolesc. Ment. Health. doi: 10.111/ camh.12375.

\section{References:}

${ }^{1}$ Punukollu, M. et al. (2019), Effectiveness of a pilot schoolbased intervention on improving Scottish students' mental health: a mixed methods evaluation. Int. J. Adolesc. Youth. 25:505-518.doi: $10.1080 / 02673843.2019 .1674167$.

See also:

https://safespot.org.uk 